\title{
Erratum: Magic wavelengths for trapping the alkali-metal atoms with circularly polarized light [Phys. Rev. A 87, 023402 (2013)]
}

\author{
B. K. Sahoo and Bindiya Arora
}

(Received 11 March 2019; published 2 April 2019)

\section{DOI: 10.1103/PhysRevA.99.049902}

In the above-named work, we had investigated magic wavelengths of some of the alkali-metal atoms using linearly and circularly polarized light. The magic wavelengths using linearly polarized light reported were very accurate, but they were not correct for the circularly polarized light due to a wrong formula given by Eq. (3) in our paper. The formula misses a factor of $\frac{1}{2}$ in the vector component contribution and assumes both $\cos \left(\theta_{k}\right)$ and $\cos \left(\theta_{p}\right)$ values to be equal to 1 for the weak magnetic field. The correct formula for the dynamic polarizability of a state with valence orbital $v$ and angular frequency $\omega$ of the circularly polarized electric field [Eq. (3) of our work] is given as

$$
\begin{aligned}
\alpha_{v}(\omega)= & \alpha_{v}^{0}(\omega)+\mathcal{A} \cos \theta_{k} \frac{m_{j}}{2 j} \alpha_{v}^{1}(\omega) \\
& +\left\{\frac{3 \cos ^{2} \theta_{p}-1}{2}\right\} \frac{3 m_{j}^{2}-j(j+1)}{j(2 j-1)} \alpha_{v}^{2}(\omega),
\end{aligned}
$$

where $\mathcal{A}=1$ for the right-handed and $\mathcal{A}=-1$ for the lefthanded circularly polarized light. Other quantities in the above expression are defined in the original paper. In the absence of a magnetic field (or in a weak magnetic field), $\cos \left(\theta_{k}\right)$ and $\cos \left(\theta_{p}\right)$ are now chosen as 1 and 0 , respectively. Corrected Figs. 4-6 for circularly polarized light are reproduced here. Similarly, Tables IV and VI are reproduced here. Apart from these changes, the rest of the contents of the original paper remain unchanged.

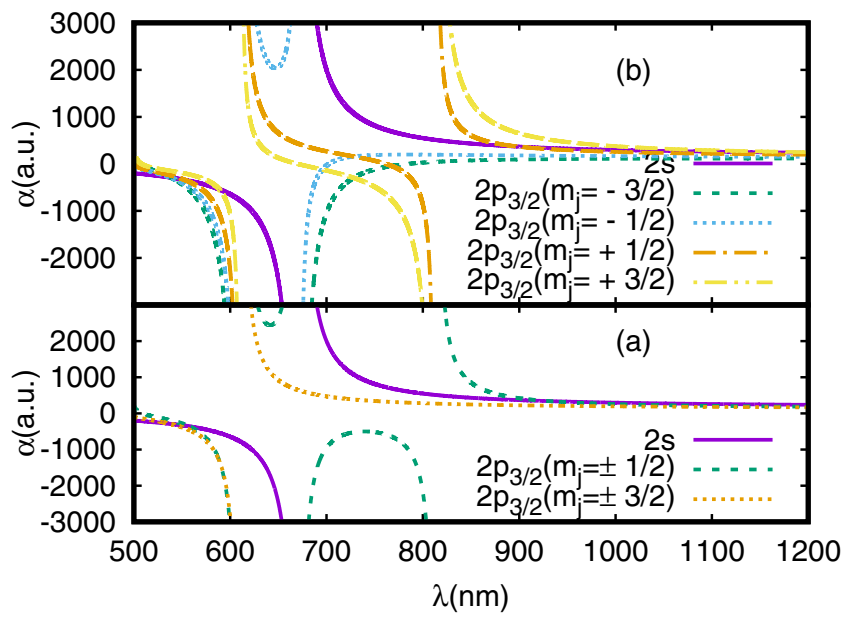

FIG. 4. Total dynamic polarizability for $2 s$ and $2 p_{3 / 2}$ states of a Li atom due to (a) linearly polarized light (b) circularly polarized light. Magic wavelength for the corresponding $2 s-2 p_{3 / 2}$ transition are given by the crossing of two polarizability curves.

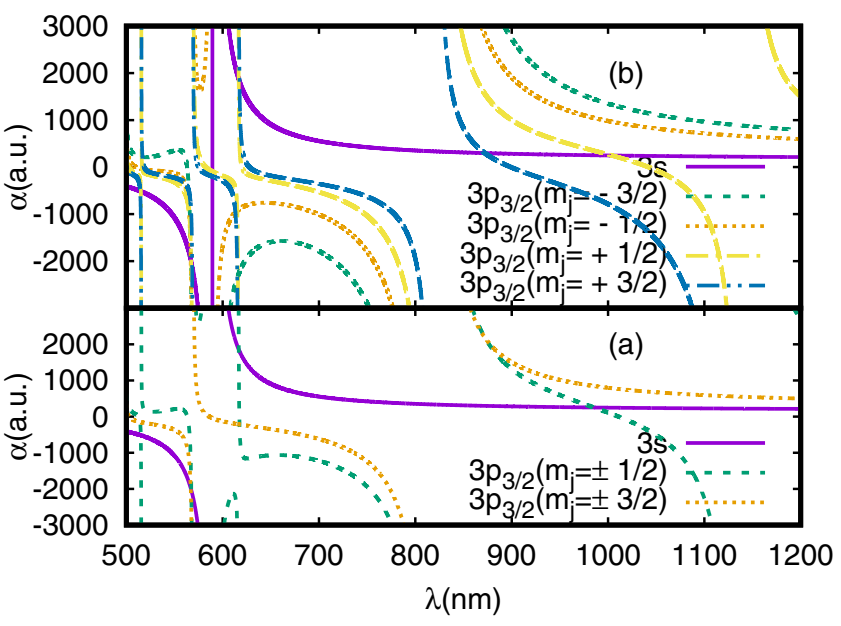

FIG. 5. Total dynamic polarizability for $3 s$ and $3 p_{3 / 2}$ states of a $\mathrm{Na}$ atom due to (a) linearly polarized light (b) circularly polarized light. Magic wavelength for the corresponding $3 s-3 p_{3 / 2}$ transition are given by the crossing of two polarizability curves.

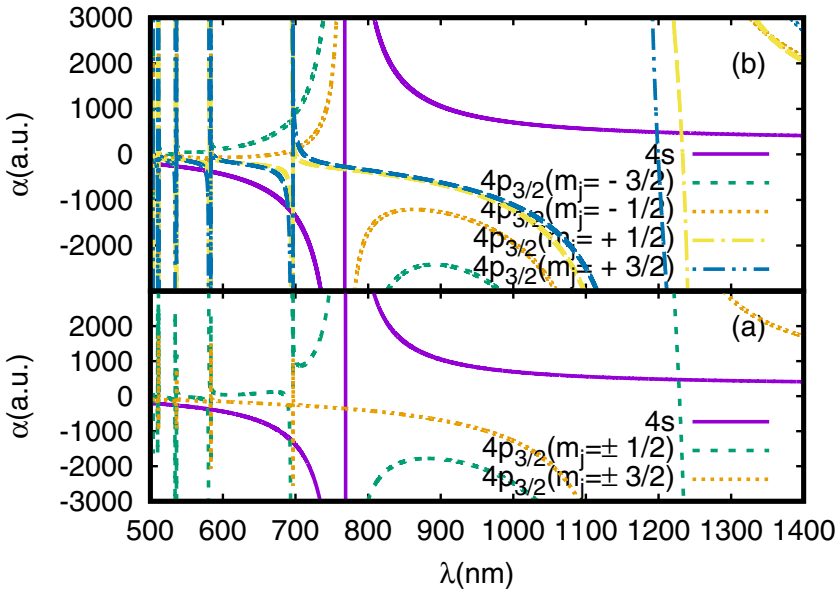

FIG. 6. Total dynamic polarizability for $4 s$ and $4 p_{3 / 2}$ states of a $\mathrm{K}$ atom due to (a) linearly polarized light (b) circularly polarized light. Magic wavelength for the corresponding $4 s-4 p_{3 / 2}$ transition are given by the crossing of two polarizability curves. 
TABLE IV. List of magic wavelengths for the circularly polarized light in $\mathrm{Li}, \mathrm{Na}$, and $\mathrm{K}$ atoms.

\begin{tabular}{|c|c|c|c|c|c|c|}
\hline \multirow[b]{2}{*}{$m_{j} \rightarrow$} & \multicolumn{2}{|c|}{$n p_{1 / 2} \rightarrow n s$} & \multicolumn{4}{|c|}{$n p_{3 / 2} \rightarrow n s$} \\
\hline & $-1 / 2$ & $1 / 2$ & $-3 / 2$ & $-1 / 2$ & $1 / 2$ & $3 / 2$ \\
\hline \multicolumn{7}{|l|}{$\mathrm{Li}$} \\
\hline$\alpha\left(\lambda_{\text {magic }}\right)$ & -292 & -422 & -292 & -290 & -340 & -540 \\
\hline$\lambda_{\text {magic }}$ & $539(2)$ & $571(1)$ & $539(1)$ & $538(2)$ & $553(2)$ & $588.7(6)$ \\
\hline$\alpha\left(\lambda_{\text {magic }}\right)$ & & 272 & & & 368 & 223 \\
\hline$\lambda_{\text {magic }}$ & & $1062(31)$ & & & $899(5)$ & $1298(66)$ \\
\hline \multicolumn{7}{|l|}{$\mathrm{Na}$} \\
\hline$\alpha\left(\lambda_{\text {magic }}\right)$ & & -509 & & & -516 & -509 \\
\hline$\lambda_{\text {magic }}$ & & $514.22(2)$ & & & $515.08(1)$ & $514.29(3)$ \\
\hline$\alpha\left(\lambda_{\text {magic }}\right)$ & -1901 & -1999 & -1960 & -1958 & -2007 & -2107 \\
\hline$\lambda_{\text {magic }}$ & $566.13(1)$ & $567.22(1)$ & $566.79(1)$ & $566.78(1)$ & $567.30(1)$ & $568.30(1)$ \\
\hline$\alpha\left(\lambda_{\text {magic }}\right)$ & & 1892 & & & 1874 & 1823 \\
\hline$\lambda_{\text {magic }}$ & & $616.356(5)$ & & & $616.629(3)$ & $617.436(5)$ \\
\hline$\alpha\left(\lambda_{\text {magic }}\right)$ & & 273 & & & 247 & 298 \\
\hline$\lambda_{\text {magic }}$ & & $923.0(5)$ & & & $1004.0(7)$ & $872.1(3)$ \\
\hline \multicolumn{7}{|l|}{ K } \\
\hline$\alpha\left(\lambda_{\text {magic }}\right)$ & -205 & -203 & -206 & -206 & -206 & -205 \\
\hline$\lambda_{\text {magic }}$ & $502.8(1)$ & $502.33(4)$ & $504.0(1)$ & $504.0(1)$ & $503.73(6)$ & $503.29(6)$ \\
\hline$\alpha\left(\lambda_{\text {magic }}\right)$ & & -211 & & & -215 & -213 \\
\hline$\lambda_{\text {magic }}$ & & $507.35(4)$ & & & $509.41(4)$ & $508.28(5)$ \\
\hline$\alpha\left(\lambda_{\text {magic }}\right)$ & -214 & -214 & -217 & -217 & -217 & -217 \\
\hline$\lambda_{\text {magic }}$ & $509.30(2)$ & $509.50(2)$ & $510.77(6)$ & $510.8(7)$ & $510.82(5)$ & $510.84(2)$ \\
\hline$\alpha\left(\lambda_{\text {magic }}\right)$ & & & -217 & -217 & -217 & -218 \\
\hline$\lambda_{\text {magic }}$ & & & $511.2(1)$ & $511.2(1)$ & $511.23(1)$ & $511.325(4)$ \\
\hline$\alpha\left(\lambda_{\text {magic }}\right)$ & & -251 & & & -256 & -253 \\
\hline$\lambda_{\text {magic }}$ & & $530.73(5)$ & & & $533.21(4)$ & $531.56(7)$ \\
\hline$\alpha\left(\lambda_{\text {magic }}\right)$ & -257 & -258 & -260 & -260 & -256 & -261 \\
\hline$\lambda_{\text {magic }}$ & $533.87(2)$ & $534.26(1)$ & $535.51(1)$ & $535.51(2)$ & $535.78(1)$ & $536.01(1)$ \\
\hline$\alpha\left(\lambda_{\text {magic }}\right)$ & & -355 & & & -365 & -356 \\
\hline$\lambda_{\text {magic }}$ & & $575.58(5)$ & & & $578.94(4)$ & $576.13(8)$ \\
\hline$\alpha\left(\lambda_{\text {magic }}\right)$ & -371 & -372 & -377 & -377 & -377 & -378 \\
\hline$\lambda_{\text {magic }}$ & $581.03(1)$ & $581.27(1)$ & $582.97(1)$ & $582.97(1)$ & $583.14(1)$ & $583.29(1)$ \\
\hline$\alpha\left(\lambda_{\text {magic }}\right)$ & & 1134 & & & -1213 & -1156 \\
\hline$\lambda_{\text {magic }}$ & & $688.08(5)$ & & & $692.54(4)$ & $689.38(4)$ \\
\hline$\alpha\left(\lambda_{\text {magic }}\right)$ & -1236 & -1237 & -1293 & -1293 & -1293 & -1293 \\
\hline$\lambda_{\text {magic }}$ & $693.740(3)$ & $693.800(2)$ & $696.60(1)$ & $696(2)$ & $696.60(5)$ & $696.61(5)$ \\
\hline$\alpha\left(\lambda_{\text {magic }}\right)$ & 26080 & -303 & & & -327 & -271 \\
\hline$\lambda_{\text {magic }}$ & $767.39(5)$ & $767.84(1)$ & & & $767.84(1)$ & $767.84(1)$ \\
\hline$\alpha\left(\lambda_{\text {magic }}\right)$ & & 485 & & & 472 & 489 \\
\hline$\lambda_{\text {magic }}$ & & $1205.1(5)$ & & & $1231.6(5)$ & 1197(1) \\
\hline
\end{tabular}


TABLE VI. List of average magic wavelengths $\lambda_{\text {magic }}(\mathrm{avg})$ for $\mathrm{Li}, \mathrm{Na}$, and $\mathrm{K}$ atoms for the leftcircularly polarized light.

\begin{tabular}{ccc}
\hline \hline & $n p_{1 / 2} \rightarrow n s$ & $n p_{3 / 2} \rightarrow n s$ \\
\hline $\mathrm{Li}$ & $555(32)$ & \\
& $1062(31)$ & $555(51)$ \\
$\mathrm{Na}$ & $514.22(2)$ & $514.7(8)$ \\
& $566.7(1)$ & $567(2)$ \\
& $616.356(5)$ & $617(1)$ \\
& $923.0(5)$ & $938(132)$ \\
$\mathrm{K}$ & $502.5(5)$ & $504(1)$ \\
& $507.35(4)$ & $509(1)$ \\
& $509.4(2)$ & $510.8(1)$ \\
& $511.2(1)$ \\
& $534(1)$ & $536(1)$ \\
& $577.58(5)$ & $577(3)$ \\
& $688.08(5)$ & $691(4)$ \\
& $688(4)$ & $767.84(1)$ \\
& $767.6(4)$ & $1214(34)$ \\
\hline \hline
\end{tabular}

